\title{
Escherichia coli
}

National Cancer Institute

\section{Source}

National Cancer Institute. Escherichia coli. NCI Thesaurus. Code C14206.

A common, gram negative gut bacterium that has been studied intensively by geneticists because of its small genome size, normal lack of pathogenicity, and ease of growth in the laboratory. 